\title{
Weyl Double Copy for Gravitational Waves
}

\author{
Hadi Godazgar* \\ Max-Planck-Institut für Gravitationsphysik (Albert-Einstein-Institut), Mühlenberg 1, D-14476 Potsdam, Germany \\ Mahdi Godazgar- ${ }^{\dagger}$ \\ School of Mathematical Sciences, Queen Mary University of London, Mile End Road, London E1 4NS, United Kingdom \\ Ricardo Monteiro and David Peinador Veiga $\odot^{\S}$ \\ Centre for Research in String Theory, School of Physics and Astronomy, Queen Mary University of London, \\ E1 4NS, United Kingdom \\ C. N. Pope $\mathrm{A}^{4, \|}$ \\ George P. \& Cynthia Woods Mitchell Institute for Fundamental Physics and Astronomy, Texas A\&M University, \\ College Station, Texas 77843, USA
}

(Received 20 October 2020; revised 21 December 2020; accepted 17 February 2021; published 11 March 2021)

\begin{abstract}
We establish the status of the Weyl double copy relation for radiative solutions of the vacuum Einstein equations. We show that all type $N$ vacuum solutions, which describe the radiation region of isolated gravitational systems with appropriate falloff for the matter fields, admit a degenerate Maxwell field that squares to give the Weyl tensor. The converse statement also holds, i.e., if there exists a degenerate Maxwell field on a curved background, then the background is type $N$. This relation defines a scalar that satisfies the wave equation on the background. We show that for nontwisting radiative solutions, the Maxwell field and the scalar also satisfy the Maxwell equation and the wave equation on Minkowski spacetime. Hence, nontwisting solutions have a straightforward double copy interpretation.
\end{abstract}

DOI: 10.1103/PhysRevLett.126.101103

The discovery of gravitational waves [1] one-hundred years after Einstein formulated his general theory of relativity has led to an exciting new area of gravitational physics with possible important prospects for observational astrophysics; a development that has been anticipated eagerly for half a century [2]. An important theoretical breakthrough in this direction will include an efficient and cost-effective method of generating gravitational wave templates; waveforms computed from the theory to be compared with observed waveforms [3,4]; see Refs. [5-10] for recent reviews. Among the myriad approaches proposed to facilitate the easier and less time-consuming generation of templates is one $[11,12]$ based on techniques adapted from string theory and supergravity scattering amplitude calculations, in particular the double copy method [13-16], which describes gravitational amplitudes as a kind of inner product of gauge theory amplitudes (hence "double copy").

While initially found at the level of scattering amplitude relations, the double copy also exists at the level of classical

Published by the American Physical Society under the terms of the Creative Commons Attribution 4.0 International license. Further distribution of this work must maintain attribution to the author(s) and the published article's title, journal citation, and DOI. Funded by SCOAP ${ }^{3}$. solutions, including beyond perturbation theory for certain classes of spacetimes. One class of solutions for which a double copy relation exists is (multi) Kerr-Schild solutions, which can be thought of as exact perturbative (around Minkowski) gravitational solutions [17,18]. The correspondence between the double copy relations for scattering amplitudes and for classical solutions has been verified in various works [19-26]; see Refs. [27,28] for earlier ideas in this direction. Of particular interest in the present Letter is the Weyl double copy relation that exists for vacuum type $D$ solutions and $p p$ waves [29-31]. This relation is best expressed in spinor language [32]. In the type $D$ case, it can be shown that the Weyl spinor $\Psi_{A B C D}=$ $\left(-2 \Phi^{2}\right)^{-1 / 4} \Phi_{(A B} \Phi_{C D)}$ with $\Phi_{A B}$ a nondegenerate Maxwell spinor and $\Phi^{2} \equiv \Phi^{A B} \Phi_{A B}$. Of particular significance is the fact that the Maxwell spinor also solves the Maxwell equation on Minkowski spacetime [31]. Furthermore, $\Phi^{1 / 2}$ solves the wave equation on Minkowski spacetime. What lies behind these relations is the existence of the wellknown hidden symmetry for type $D$ vacuum solutions as expressed by the existence of a Killing 2-spinor [29,33]. See Refs. [34-49] for related works.

In this Letter, we extend the curved Weyl double copy relation to all type $N$ vacuum solutions, which describe the radiation region of isolated gravitational systems. In particular, we show that $\Psi_{A B C D}=S^{-1} \Phi_{(A B} \Phi_{C D)}$ with $\Phi_{A B}$ 
a degenerate Maxwell spinor and $S$ some scalar that, in particular, satisfies the wave equation on the curved background. For nontwisting radiative spacetimes, the Maxwell field and the scalar field also solve the Maxwell equation and the wave equation, respectively, on Minkowski spacetime. This establishes the Weyl double copy in the sense of Ref. [31] for this large class of spacetimes. Notice that, while the double copy for scattering amplitudes involves two copies of non-Abelian gauge theory, the first step in that procedure is to consider the double copy of the asymptotic states, which for linearized gauge theory are solutions to the Maxwell equation. The fact that certain exact gravity solutions can be interpreted as a double copy of a Maxwell field means that they should be interpreted as coherent states, an exact extension of the linearized asymptotic states in scattering amplitudes. For twisting spacetimes, the Maxwell field and the scalar depend generically on the metric functions. Hence, they are solutions only on the curved spacetime. However, the standard double copy interpretation applies at the linearized level. This may be indicative of the fact that twisting solutions have an intrinsic non-Abelian nature.

As a necessary step in extending the exact classical double copy tools to gravitational wave physics, we provide a systematic understanding of the status of the double copy for radiative solutions, beyond the most special example of $p p$ waves. This study reveals interesting differences with the Weyl double copy for type $D$ solutions. In particular, the construction does not lead to a unique Maxwell field, since there is functional freedom associated with the scalar $S$. In the cases where the Maxwell field can be thought of as living in Minkowski spacetime, i.e., for nontwisting solutions, it would be interesting to use novel approaches, e.g., Refs. [50-52], to relate this new construction to the double copy for scattering amplitudes, as has been done for certain type $D$ solutions.

Spinor calculus.-The homomorphism between the Lorentz group and $\mathrm{SL}(2, \mathbb{C})$ can be used to convert spacetime indices $\mu, \nu, \ldots$ into spinor $A, B, \ldots=\{1,2\}$ and conjugate spinor $\dot{A}, \dot{B}, \ldots=\{1,2\}$ indices, using the Van der Waerden matrices $\sigma_{A \dot{A}}^{\mu}$, which are constructed from the identity and Pauli matrices. It is convenient to work in a spinor basis $\left\{o^{A}, l^{A}\right\}$ with $\epsilon_{A B} o^{A} t^{B}=1$. In this basis, $\epsilon_{A B}=2 o_{\left[{ }^{A} l_{B]}\right]}$ and can be used to lower indices $\psi_{A}=\psi^{B} \epsilon_{B A}$. Similarly, $\psi^{A}=\epsilon^{A B} \psi_{B}$. Associated with the spin basis is a null frame $(\ell, n, m, \bar{m})$ [53], so that

$$
g_{\mu \nu}=2 \ell_{(\mu} n_{\nu)}+2 m_{(\mu} \bar{m}_{\nu)}
$$

Our notation follows Ref. [54]. For a vacuum spacetime, the curvature is given by the Weyl tensor. Its spinorial version is fully determined by the totally symmetric Weyl spinor $\Psi_{A B C D}$ (and its complex conjugate), which satisfies the Bianchi identity

$$
\nabla^{A \dot{A}} \Psi_{A B C D}=0
$$

Similarly, a solution of the Maxwell equation can be written in terms of a symmetric 2-spinor $\Phi_{A B}$ that solves

$$
\nabla^{A \dot{A}} \Phi_{A B}=0 .
$$

For type $N$ solutions, choosing a spinor basis adapted to the principal null direction (PND) $\ell^{\mu} \sim o_{A} \bar{o}_{\dot{A}}$, the NewmanPenrose (NP) Weyl scalars, which correspond to various components of the Weyl spinor in the spinor basis, all vanish except $\Psi_{4}=\Psi_{A B C D} l^{A} l^{B} l_{l} l^{D}=n^{\mu} \bar{m}^{\nu} n^{\rho} \bar{m}^{\sigma} C_{\mu \nu \rho \sigma}$ and the Weyl spinor takes the simple form

$$
\Psi_{A B C D}=\Psi_{4} o_{A} o_{B} o_{C} o_{D}
$$

Weyl double copy.-In spinor language, the curved background Weyl double copy relation is

$$
\Psi_{A B C D}=\frac{1}{S} \Phi_{(A B} \Phi_{C D)},
$$

for some scalar $S$ and Maxwell spinor $\Phi_{A B}$. Note that $\Phi_{A B}$ satisfies the Maxwell equation (3) in the fixed curved background metric, but it is viewed as a test field that does not backreact on the geometry. From Eq. (4), it follows that the NP Maxwell scalars all vanish except $\Phi_{2}$, and we have $\left.\Phi_{A B}=\Phi_{2} o_{(A} o_{B}\right)$. Thus the type $N$ double copy relation is

$$
\Psi_{4}=\frac{1}{S}\left(\Phi_{2}\right)^{2} .
$$

The Maxwell 2-spinor is degenerate, which means that the electromagnetic field is null, i.e., the electric and magnetic fields are perpendicular and of equal magnitude. An example of a null electromagnetic field is that of a plane electromagnetic wave in flat spacetime. Now we must consider whether such a relation [14] exists. Expanding out the Bianchi identity (2) by substituting (4) gives two equations:

$$
o_{A} \nabla^{A \dot{A}} \log \Psi_{4}+4 o_{A} l^{B} \nabla^{A \dot{A}} o_{B}-l_{A} o^{B} \nabla^{A \dot{A}} o_{B}=0
$$

and $o_{A} o^{B} \nabla^{A \dot{A}} o_{B}=0$. The second equation is equivalent to the statement that the null congruence generated by the PND is geodesic, $\kappa=0$, and shear-free, $\sigma=0$ [55], which follow from the Goldberg-Sachs theorem [56]. Expanding out the Maxwell equation in a similar fashion gives

$$
o_{A} \nabla^{A \dot{A}} \log \Phi_{2}+2 o_{A} l^{B} \nabla^{A \dot{A}} o_{B}-l_{A} o^{B} \nabla^{A \dot{A}} o_{B}=0,
$$

as well as the same equation above that is equivalent to $\kappa=\sigma=0$. Now, substituting $\Psi_{4}=\left(\Phi_{2}\right)^{2} / S$ into (7) and simplifying this using (8) gives 


$$
o_{A} \nabla^{A \dot{A}} \log S-l_{A} o^{B} \nabla^{A \dot{A}} o_{B}=0 .
$$

There is a clear structure in Eqs. (7)-(9), where the coefficient of the middle term is the rank of the respective spinor. Equation (9) translates, using $\bar{o}_{\dot{A}}$ and $\bar{l}_{\dot{A}}$, to

$$
\ell \cdot \nabla \log S-\rho=0, \quad m \cdot \nabla \log S-\tau=0,
$$

where $\rho$ and $\tau$ are NP spin coefficients [57]. $\rho$ parametrizes the expansion and twist of the null congruence generated by $\ell$, while $\tau$ parametrizes the transport of $\ell$ along the flow generated by $n$. A simple calculation shows that the integrability condition on Eqs. (10) is satisfied, which means that they are simple integral equations that can always be solved. Thus, we are guaranteed the existence of a scalar $S$ satisfying these equations, which then gives a Maxwell field $\Phi_{2}=\sqrt{\Psi_{4} S}$. In tensor language, this Maxwell spinor translates to a field strength (called the "single copy") of the form

$$
F=\Phi_{2} \ell^{b} \wedge m^{b}+\bar{\Phi}_{2} \ell^{b} \wedge \bar{m}^{b}
$$

where $\ell^{b}$ denotes the 1 -form $\ell^{b}=\ell_{\mu} d x^{\mu}$, and similarly for $m^{b}$ and $\bar{m}^{b}$. This establishes the curved Weyl double copy for type $N$ vacuum solutions.

Furthermore, it is simple to show using Eq. (9) that $S$ solves the wave equation

$$
\square S=\nabla_{A \dot{A}} \nabla^{A \dot{A}} S=2 o_{A} l_{B} \nabla^{A} \dot{A} \nabla^{B \dot{A}} S=0 .
$$

The real scalar field in the double copy construction (called the "zeroth copy") is the real part of $S$.

These results mirror those that exist for type $D$ solutions. In order to investigate whether the Maxwell field and the scalar field also satisfy the equations of motion on Minkowski spacetime, we investigate the different classes of type $N$ solutions in turn.

Type $N$ vacuum solutions.-Type $N$ vacuum solutions are classified in terms of the optical properties of the congruence generated by the PND, i.e., by the values of the optical scalars; see, e.g., Ref. [54]. We have $\kappa=\sigma=0$, as mentioned before; the properties that remain are parametrized by the spin coefficient $\rho=-(\Theta+i \omega)$, where $\Theta$ denotes the expansion of the congruence and $\omega$ denotes its twist. The different cases lead to three distinct classes of solutions: (i) Kundt solutions: $\Theta=0$, which implies that $\omega=0$ [58]. (ii) Robinson-Trautman solutions: $\Theta \neq 0$, $\omega=0$. (iii) Twisting solutions: $\Theta \neq 0, \omega \neq 0$. Choosing a null frame for which $\ell$ is the PND, so that $\Psi_{0}=\Psi_{1}=$ $\Psi_{2}=\Psi_{3}=0$, we consider each case separately.

Kundt solutions.-There are two kinds of type $N$ Kundt solutions, both corresponding to plane-fronted wave solutions [59]. Plane-fronted waves with parallel propagation ( $p p$ waves) are given by the metric

$$
d s^{2}=-2 d u(d v+H d u)+2 d z d \bar{z}
$$

with $H(u, z, \bar{z})=f(u, z)+\bar{f}(u, \bar{z})$ for general functions $f$. Choosing

$$
\ell=\partial_{v}, \quad n=\partial_{u}-H \partial_{v}, \quad m=\partial_{z},
$$

one has $\rho=\tau=0$ and so Eq. (10) implies $S=S(u, \bar{z})$, while the Weyl scalar $\Psi_{4}=\partial_{\bar{z}}^{2} \bar{f}$, so Eq. (6) implies that

$$
\Phi_{2}=\sqrt{\partial_{\bar{z}}^{2} \bar{f} S(u, \bar{z})} .
$$

The other class of plane-fronted waves is given by

$d s^{2}=-2 d u(d v+W d z+\bar{W} d \bar{z}+H d u)+2 d z d \bar{z}$,

with $W(v, z, \bar{z})=-2 v(z+\bar{z})^{-1}$ and

$$
H(u, v, z, \bar{z})=[f(u, z)+\bar{f}(u, \bar{z})](z+\bar{z})-\frac{v^{2}}{(z+\bar{z})^{2}} ;
$$

again $f(u, z)$ is arbitrary. Choosing

$$
\ell=\partial_{v}, n=\partial_{u}-(H+W \bar{W}) \partial_{v}+\bar{W} \partial_{z}+W \partial_{\bar{z}}, m=\partial_{z},
$$

one has $\rho=0, \tau=2 \beta=-(z+\bar{z})^{-1}$, so Eq. (10) gives $S=\zeta(u, \bar{z}) /(z+\bar{z})$. The Weyl scalar $\Psi_{4}=(z+\bar{z}) \partial_{\bar{z}}^{2} \bar{f}$, so Eq. (6) implies that

$$
\Phi_{2}=\sqrt{\partial_{\bar{z}}^{2} \bar{f} \zeta(u, \bar{z})} .
$$

Given that the only nonzero components of $F_{\mu \nu}$ are for $\mu \nu=[u z]$ and $[u \bar{z}]$, the simple form of the relevant components of $g^{\mu \nu}$ and the fact that $g=1$ give

$$
\begin{array}{r}
\nabla_{\nu} F^{\mu \nu}=\frac{1}{\sqrt{|g|}} \partial_{\nu}\left(\sqrt{|g|} g^{\mu \rho} g^{\nu \sigma} F_{\rho \sigma}\right) \\
\quad=\partial_{\nu}\left(\eta^{\mu \rho} \eta^{\nu \sigma} F_{\rho \sigma}\right)=0 .
\end{array}
$$

On the other hand, $S$ does not depend on $f(u, z)$ or $\bar{f}(u, \bar{z})$, meaning that it must solve the wave equation on any member of the family. In particular, it solves the wave equation on Minkowski spacetime. This implies that the Maxwell and the scalar fields also satisfy their equations on Minkowski spacetime, establishing the Weyl double copy for type $N$ Kundt solutions.

Robinson-Trautman solutions.-Type $N$ RobinsonTrautman solutions take the form [60]

$$
d s^{2}=-H d u^{2}-2 d u d r+\frac{2 r^{2}}{P^{2}} d z d \bar{z},
$$

with $H(u, r, z, \bar{z})=k-2 r \partial_{u} \log P($ where $k=0, \pm 1)$ and $2 P^{2} \partial_{z} \partial_{\bar{z}} \log P(u, z, \bar{z})=k$. Choosing 


$$
\ell=\partial_{r}, \quad n=\partial_{u}-\frac{1}{2} H \partial_{r}, \quad m=-\frac{P}{r} \partial_{z}
$$

one has $\rho=-r^{-1}, \tau=0$, so Eq. (10) gives $S=-\zeta(u, \bar{z}) / r$. Now $\Psi_{4}=-\left(P^{2} / r\right) \partial_{u}\left(\partial_{\bar{z}}^{2} P / P\right)$, so Eq. (6) determines that

$$
\Phi_{2}=\frac{P}{r} \sqrt{\zeta(u, \bar{z}) \partial_{u}\left(\partial_{\bar{z}}^{2} P / P\right)} .
$$

As an example, consider Robinson-Trautman solutions with $k=0$ in Eq. (19). Writing $P=e^{W}$ we have $\partial_{z} \partial_{\bar{z}} W=$ 0 and hence $W=w(u, z)+\bar{w}(u, \bar{z})$, implying that $\Psi_{4}=-P^{2} / r \partial_{u}\left[\partial_{\bar{z}}^{2} \bar{w}(u, \bar{z})+\left(\partial_{\bar{z}} \bar{w}(u, \bar{z})^{2}\right]\right.$. We can obtain type $N$ solutions of the Maxwell equation in the Robinson-Trautman background by taking

$$
A=\gamma(u, z, \bar{z}) d u,
$$

where $\partial_{z} \partial_{\bar{z}} \gamma=0$ and hence $\gamma=h(u, z)+\bar{h}(u, \bar{z})$. Thus from Eq. (11) we have $\Phi_{2}=-P / r \partial_{\bar{z}} \bar{h}(u, \bar{z})$. Plugging into Eq. (6) we have

$$
\partial_{u}\left[\partial_{\bar{z}}^{2} \bar{w}(u, \bar{z})+\left[\partial_{\bar{z}} \bar{w}(u, \bar{z})\right]^{2}\right]=-\frac{1}{r S}\left[\partial_{\bar{z}} \bar{h}(u, \bar{z})\right]^{2},
$$

and so indeed we have that $S=-\zeta / r$, where $\zeta$ is a function only of $u$ and $\bar{z}$, as required in the general result stated above.

As with Kundt solutions, the only nonzero components of $F_{\mu \nu}$ are for $\mu \nu=[u z]$ and $[u \bar{z}]$. As before, using the fact that $\sqrt{|g|}=r^{2} / P^{2}$ and the relevant components of $g^{\mu \nu}$, it can be shown that Eq. (18) holds. Once again, $S$ is independent of $P$ and solves the wave equation on any member of the family (19), including Minkowski. Hence, both $F_{\mu \nu}$ and $S$ satisfy their equations also on the flat background, establishing the Weyl double copy for Robinson-Trautman solutions.

Twisting solutions.-Type $N$ solutions with nonvanishing twist are more complicated, with only one explicit solution known [61]. The general metric is given by [62]

$$
\begin{aligned}
d s^{2}= & -2(d u+L d z+\bar{L} d \bar{z})[d r+W d z+\bar{W} d \bar{z} \\
& +H(d u+L d z+\bar{L} d \bar{z})]+\frac{2}{P^{2}|\rho|^{2}} d z d \bar{z}, \\
\rho^{-1}= & -(r+i \Sigma), \quad 2 i \Sigma(u, z, \bar{z})=P^{2}(\bar{\partial} L-\partial \bar{L}), \\
W(u, r, z, \bar{z})= & \rho^{-1} \partial_{u} L+i \partial \Sigma, \quad \partial=\partial_{z}-L \partial_{u}, \\
H(u, r, z, \bar{z})= & \frac{1}{2} K-r \partial_{u} \log P,
\end{aligned}
$$

with $K=2 P^{2} \Re\left[\partial\left(\bar{\partial} \log P-\partial_{u} \bar{L}\right)\right]$. There exists a residual gauge freedom to choose $P=1$, but we shall not yet impose this choice. The solution is determined by the complex scalar $L$, which satisfies
$\Sigma K+P^{2} \Re\left[\partial \bar{\partial} \Sigma-2 \partial_{u} \bar{L} \partial \Sigma-\Sigma \partial_{u} \partial \bar{L}\right]=0, \quad \partial I=0$,

and $\partial_{u} I \neq 0$, with $I=\bar{\partial}\left(\bar{\partial} \log P-\partial_{u} \bar{L}\right)+\left(\bar{\partial} \log P-\partial_{u} \bar{L}\right)^{2}$. Choosing

$$
\ell=\partial_{r}, \quad n=\partial_{u}-H \partial_{r}, \quad m=-P \bar{\rho}\left(\partial-W \partial_{r}\right),
$$

$\rho$ is as defined above, while $\tau=0$. Equation (10) then implies that $S=\rho \chi(u, z, \bar{z})$, with $\chi$ satisfying

$$
\partial \chi-\partial_{u} L \chi=0
$$

Defining new coordinates $(v, w)=(I, z)$, the above equation can be solved using the method of characteristics ( $I=$ constant correspond to the characteristics $)$

$$
\chi(v, w)=\zeta(I) e^{\left.\int\left[\left(\frac{\partial I(u, z)}{\partial u}\right)\right)\left(v, w^{\prime}\right) \times \frac{\partial L\left(v, w^{\prime}\right)}{\partial v}\right] d w^{\prime}},
$$

with $\zeta(I)$ arbitrary. The Weyl scalar $\Psi_{4}=\rho P^{2} \partial_{u} I$, and so Eq. (6) implies

$$
\Phi_{2}=\rho P \sqrt{\partial_{u} I \chi(u, z, \bar{z})} .
$$

Only one twisting type $N$ solution, found by Hauser [61], is known explicitly. The metric functions are given by

$$
P=(z+\bar{z})^{3 / 2} f(t), \quad t \equiv \frac{u}{(z+\bar{z})^{2}}, \quad L=2 i(z+\bar{z}),
$$

where $f$ satisfies $16\left(1+t^{2}\right) f^{\prime \prime}(t)+3 f(t)=0$, which is a hypergeometric equation, and $I$ turns out to be given by

$$
I=\frac{3}{2\left[(z+\bar{z})^{2}-i u\right]} .
$$

The solution to Eq. (10) is

$$
S=\rho \zeta(I)
$$

where $\zeta(I)$ is arbitrary. As expected, this is consistent with the general result (27). The Weyl scalar is $\Psi_{4}=$ $(2 i / 3) \rho P^{2} I^{2}$, implying that

$$
\Phi_{2}=\rho P I \sqrt{\frac{2 i \zeta(I)}{3}} .
$$

As a further remark about the twisting type $N$ solutions, we note that if the gauge freedom to set $P=1$ is employed, the metric is specified purely in terms of the function $L(u, z, \bar{z})$, and the type $N$ and Ricci flat conditions may be succinctly condensed down to just

$\partial I=0, \quad \Im(\bar{\partial} \bar{\partial} \partial \partial L)=0, \quad$ where $I=-\partial_{u} \bar{\partial} \bar{L}$.

The Weyl curvature is given by $\Psi_{4}=\rho \partial_{u} I$. 
In contrast to nontwisting solutions, the second equality in Eq. (18) does not hold for twisting solutions. Therefore, while there is a curved Weyl double copy relation, in this case it does not translate to a relation where the Maxwell field and the scalar can be thought of as Minkowski fields, unless we consider all the fields (gravity, Maxwell, and scalar) at the linearized level.

Nonuniqueness. - In all the cases above, neither the Maxwell field nor the scalar field are uniquely determined. They are fixed only up to an arbitrary function of some of the coordinates, which we are free to choose. This contrasts with the Weyl double copy for vacuum type $D$ solutions, for which, in a spinor basis adapted to the principal null directions, we have $S^{3} \propto\left(\Phi_{2}\right)^{3 / 2} \propto \Psi_{4}$, where the proportionality is up to complex parameters [31]; hence the Maxwell and scalar fields are functionally fixed. This feature is related to the fact that vacuum type $D$ spacetimes are fully determined up to a few parameters, whereas vacuum type $\mathrm{N}$ spacetimes (of any class, as seen above) have functional freedom. By analogy, there is additional freedom in the Maxwell and scalar fields in the curved background.

In considering a special choice, we may ask whether it is possible to choose $\Phi_{2}$ and $S$ to be given by specific powers of $\Psi_{4}$, as in the type $D$ case, i.e., there exists some constant $a$ such that $\Phi_{2} \propto\left(\Psi_{4}\right)^{a}$ and $S \propto\left(\Psi_{4}\right)^{2 a-1}$. The functional dependence of the results above implies that this possibility holds only for Kundt solutions. For $p p$ waves, the power is actually undetermined, i.e., the relation above holds for any $a$. A simple choice is $a=1 / 2$, where $S$ is constant, and in fact this choice implies that Maxwell plane waves double copy to gravitational plane waves $\left(\Phi_{2}\right.$ and $\Psi_{4}$ are functions of $u$ only). For the other plane-fronted Kundt solutions, such a relation is possible for $a=0$, in which case $S \propto\left(\Psi_{4}\right)^{-1}$. Analogously simple choices for the other type $N$ classes are $S \propto 1 / r$ for Robinson-Trautman solutions and $S \propto \rho$ for twisting solutions.

Interestingly, $p p$ waves are the only type $N$ solutions admitting a Killing 2-spinor [30], another feature that they share with type $D$ solutions.

A twistorial version of the Weyl double copy is given in Ref. [63], focusing on type $D$ but also introducing some type III cases, at least at the linearized level. It would be interesting to study whether this twistorial version explains the nonuniqueness of the type $N$ Weyl double copy found here.

We would like to thank Donal O'Connell and Chris White for discussions. H. G. would like to thank Queen Mary University of London for hospitality during the course of this work. H.G. is supported by the ERC Advanced Grant Exceptional Quantum Gravity (Grant No. 740209). M. G. and R. M. are supported by Royal Society University Research Fellowships. C. N. P. is partially supported by DOE Grant No. DE-FG02-13ER42020. D. P. V. is supported by a Royal Society studentship grant. *hadi.godazgar@aei.mpg.de

†m.godazgar@qmul.ac.uk

Fricardo.monteiro@qmul.ac.uk

§.peinadorveiga@qmul.ac.uk

" pope@physics.tamu.edu

Also at Centre for Mathematical Sciences, Cambridge University, Wilberforce Road, Cambridge, CB3 0WA, UK.

[1] B. P. Abbott et al. (LIGO Scientific and Virgo Collaborations), Observation of Gravitational Waves from a Binary Black Hole Merger, Phys. Rev. Lett. 116, 061102 (2016).

[2] W.H. Press and K.S. Thorne, Gravitational-wave astronomy, Annu. Rev. Astron. Astrophys. 10, 335 (1972).

[3] A. Buonanno and T. Damour, Effective one-body approach to general relativistic two-body dynamics, Phys. Rev. D 59, 084006 (1999).

[4] F. Pretorius, Evolution of Binary Black Hole Spacetimes, Phys. Rev. Lett. 95, 121101 (2005).

[5] M. Shibata and K. Taniguchi, Coalescence of black holeneutron star binaries, Living Rev. Relativity 14, 6 (2011).

[6] N. T. Bishop and L. Rezzolla, Extraction of gravitational waves in numerical relativity, Living Rev. Relativity 19, 2 (2016).

[7] L. Blanchet, Gravitational radiation from post-Newtonian sources and inspiralling compact binaries, Living Rev. Relativity 17, 2 (2014).

[8] R. A. Porto, The effective field theorist's approach to gravitational dynamics, Phys. Rep. 633, 1 (2016).

[9] L. Barack and A. Pound, Self-force and radiation reaction in general relativity, Rep. Prog. Phys. 82, 016904 (2019).

[10] M. Levi, Effective field theories of post-Newtonian gravity: A comprehensive review, Rep. Prog. Phys. 83, 075901 (2020).

[11] Z. Bern, C. Cheung, R. Roiban, C.-H. Shen, M. P. Solon, and M. Zeng, Scattering Amplitudes and the Conservative Hamiltonian for Binary Systems at Third Post-Minkowskian Order, Phys. Rev. Lett. 122, 201603 (2019).

[12] Z. Bern, C. Cheung, R. Roiban, C.-H. Shen, M. P. Solon, and M. Zeng, Black hole binary dynamics from the double copy and effective theory, J. High Energy Phys. 10 (2019) 206.

[13] H. Kawai, D. Lewellen, and S. Tye, A relation between tree amplitudes of closed and open strings, Nucl. Phys. B269, 1 (1986).

[14] Z. Bern, J. J. M. Carrasco, and H. Johansson, New relations for gauge-theory amplitudes, Phys. Rev. D 78, 085011 (2008).

[15] Z. Bern, John Joseph M. Carrasco, and H. Johansson, Perturbative Quantum Gravity as a Double Copy of Gauge Theory, Phys. Rev. Lett. 105, 061602 (2010).

[16] Z. Bern, J. J. Carrasco, M. Chiodaroli, H. Johansson, and R. Roiban, The duality between color and kinematics and its applications, arXiv:1909.01358.

[17] R. Monteiro, D. O'Connell, and C. D. White, Black holes and the double copy, J. High Energy Phys. 12 (2014) 056.

[18] A. Luna, R. Monteiro, D. O'Connell, and C. D. White, The classical double copy for Taub-NUT spacetime, Phys. Lett. B 750, 272 (2015).

[19] A. Luna, R. Monteiro, I. Nicholson, D. O'Connell, and C. D. White, The double copy: Bremsstrahlung and accelerating black holes, J. High Energy Phys. 06 (2016) 023. 
[20] W. D. Goldberger and A. K. Ridgway, Radiation and the classical double copy for color charges, Phys. Rev. D 95, 125010 (2017).

[21] A. Luna, R. Monteiro, I. Nicholson, A. Ochirov, D. O'Connell, N. Westerberg, and C. D. White, Perturbative spacetimes from Yang-Mills theory, J. High Energy Phys. 04 (2017) 069.

[22] N. Arkani-Hamed, Y.-t. Huang, and D. O'Connell, Kerr black holes as elementary particles, J. High Energy Phys. 01 (2020) 046.

[23] Y.-T. Huang, U. Kol, and D. O'Connell, Double copy of electric-magnetic duality, Phys. Rev. D 102, 046005 (2020).

[24] K. Kim, K. Lee, R. Monteiro, I. Nicholson, and D. Peinador Veiga, The classical double copy of a point charge, J. High Energy Phys. 02 (2020) 046.

[25] A. Luna, S. Nagy, and C. White, The convolutional double copy: A case study with a point, J. High Energy Phys. 09 (2020) 062.

[26] A. Cristofoli, Gravitational shock waves and scattering amplitudes, J. High Energy Phys. 11 (2020) 160.

[27] R. Saotome and R. Akhoury, Relationship between gravity and gauge scattering in the high energy limit, J. High Energy Phys. 01 (2013) 123.

[28] D. Neill and I. Z. Rothstein, Classical space-times from the S matrix, Nucl. Phys. B877, 177 (2013).

[29] M. Walker and R. Penrose, On quadratic first integrals of the geodesic equations for type $\{22\}$ spacetimes, Commun. Math. Phys. 18, 265 (1970).

[30] W. Dietz and R. Rüdiger, Space-times admitting KillingYano tensors. I, Proc. R. Soc. A 375, 361 (1981).

[31] A. Luna, R. Monteiro, I. Nicholson, and D. O'Connell, Type D spacetimes and the Weyl double copy, Classical Quantum Gravity 36, 065003 (2019).

[32] We summarize some important equations regarding spinor calculus in curved spacetime below. For comprehensive introductions, see Refs. [64,65].

[33] B. Carter, Global structure of the Kerr family of gravitational fields, Phys. Rev. 174, 1559 (1968).

[34] M. Carrillo-González, R. Penco, and M. Trodden, The classical double copy in maximally symmetric spacetimes, J. High Energy Phys. 04 (2018) 028.

[35] A. Ilderton, Screw-symmetric gravitational waves: A double copy of the vortex, Phys. Lett. B 782, 22 (2018).

[36] K. Lee, Kerr-Schild double field theory and classical double copy, J. High Energy Phys. 10 (2018) 027.

[37] D. S. Berman, E. Chacón, A. Luna, and C. D. White, The self-dual classical double copy, and the Eguchi-Hanson instanton, J. High Energy Phys. 01 (2019) 107.

[38] K. Andrzejewski and S. Prencel, From polarized gravitational waves to analytically solvable electromagnetic beams, Phys. Rev. D 100, 045006 (2019).

[39] S. Sabharwal and J. W. Dalhuisen, Anti-self-dual spacetimes, gravitational instantons and knotted zeros of the Weyl tensor, J. High Energy Phys. 07 (2019) 004.

[40] H. Godazgar, M. Godazgar, and C. Pope, Taub-NUT from the Dirac monopole, Phys. Lett. B 798, 134938 (2019).

[41] I. Bah, R. Dempsey, and P. Weck, Kerr-Schild Double copy and complex worldlines, J. High Energy Phys. 02 (2020) 180.
[42] R. Alawadhi, D. S. Berman, B. Spence, and D. Peinador Veiga, S-duality and the double copy, J. High Energy Phys. 03 (2020) 059.

[43] A. Banerjee, E. Colgáin, J. Rosabal, and H. Yavartanoo, Ehlers as EM duality in the double copy, Phys. Rev. D 102, 126017 (2020).

[44] C. Keeler, T. Manton, and N. Monga, From Navier-Stokes to Maxwell via Einstein, J. High Energy Phys. 08 (2020) 147.

[45] N. Bahjat-Abbas, R. Stark-Muchão, and C. D. White, Monopoles, shockwaves and the classical double copy, J. High Energy Phys. 04 (2020) 102.

[46] G. Elor, K. Farnsworth, M. L. Graesser, and G. Herczeg, The Newman-Penrose map and the classical double copy, J. High Energy Phys. 12 (2020) 121.

[47] R. Alawadhi, D. S. Berman, and B. Spence, Weyl doubling, J. High Energy Phys. 09 (2020) 127.

[48] L. Alfonsi, C. D. White, and S. Wikeley, Topology and Wilson lines: Global aspects of the double copy, J. High Energy Phys. 07 (2020) 091.

[49] T. Adamo and A. Ilderton, Classical and quantum double copy of back-reaction, J. High Energy Phys. 09 (2020) 200.

[50] T. Adamo, E. Casali, L. Mason, and S. Nekovar, Scattering on plane waves and the double copy, Classical Quantum Gravity 35, 015004 (2018).

[51] A. Anastasiou, L. Borsten, M. J. Duff, S. Nagy, and M. Zoccali, Gravity as Gauge Theory Squared: A Ghost Story, Phys. Rev. Lett. 121, 211601 (2018).

[52] D. A. Kosower, B. Maybee, and D. O'Connell, Amplitudes, observables, and classical scattering, J. High Energy Phys. 02 (2019) 137.

[53] The null vectors are constructed from the spinors as follows: $\ell^{\mu} \sim o_{A} \bar{o}_{\dot{A}}, n^{\mu} \sim l_{A} \bar{l}_{\dot{A}}, m^{\mu} \sim o_{A} \bar{l}_{\dot{A}}$ and $\bar{m}^{\mu} \sim l_{A} \bar{o}_{\dot{A}}$.

[54] H. Stephani, D. Kramer, M. A. H. MacCallum, C. Hoenselaers, and E. Herlt, Exact Solutions of Einstein's Field Equations, Cambridge Monographs on Mathematical Physics (Cambridge University Press, Cambridge, England, 2003).

[55] $\kappa=-\ell^{\mu} m^{\nu} \nabla_{\mu} \ell_{\nu}, \sigma=-m^{\mu} m^{\nu} \nabla_{\mu} \ell_{\nu}$.

[56] J. N. Goldberg and R. K. Sachs, A theorem on Petrov types, Acta Phys. Pol. 22, 13 (1962).

[57] $\rho=-\bar{m}^{\mu} m^{\nu} \nabla_{\mu} \ell_{\nu}, \tau=-n^{\mu} m^{\nu} \nabla_{\mu} \ell_{\nu}$.

[58] Substituting $\Theta=\sigma=R_{\mu \nu}=0$ into the Raychaudhuri equation $\ell \cdot \nabla \Theta-\omega^{2}+\Theta^{2}+\sigma \bar{\sigma}+\frac{1}{2} R_{\mu \nu} \ell^{\mu} \ell^{\nu}=0$ gives $\omega=0$.

[59] See Theorem 31.2 of Ref. [54].

[60] See Theorem 28.1 and Sec. 28.1.2 of Ref. [54].

[61] I. Hauser, Type-N Gravitational Field with Twist, Phys. Rev. Lett. 33, 1112 (1974).

[62] See Chap. 29 of Ref. [54].

[63] C. D. White, A Twistorial Foundation for the Classical Double Copy, Phys. Rev. Lett. 126, 061602 (2021).

[64] R. Penrose and W. Rindler, Spinors and Space-Time. Vol. 1: Two-Spinor Calculus and Relativistic Fields (Cambridge University Press, Cambridge, England, 1984).

[65] J. Stewart, Advanced General Relativity, Cambridge Monographs on Mathematical Physics (Cambridge University Press, Cambridge, England, 1991). 